\title{
The Impact of Aspirin Intake on Lactate Dehydrogenase, Arterial Stiffness, and Oxidative Stress During High-Intensity Exercise: A Pilot Study
}

\author{
by \\ Sang Ho Lee', Elizabeth J. Pekas², Seungyong Lee 3 , Ronald J. Headid III', \\ Song-Young Park ${ }^{2}$
}

\begin{abstract}
Aspirin is a common nonsteroidal anti-inflammatory drug used to reduce fever, pain, and inflammation. However, aspirin's anti-inflammatory properties may also prevent increased levels of blood lactate dehydrogenase, vascular arterial stiffness and oxidative stress induced by high-intensity exercise. The purpose of this study was to investigate the effects of 4 weeks of aspirin supplementation on lactate dehydrogenase activity, lactate, arterial stiffness, and antioxidant capacity during high-intensity exercise in Taekwondo athletes. Participants were randomly divided into two groups: aspirin supplementation $(n=10)$ and placebo-control $(n=10)$. Blood levels of lactate dehydrogenase $(L D H)$ enzyme activity and lactate were assessed to examine muscle damage and carotid-to-radial pulse wave velocity and the augmentation index were measured to examine arterial stiffness. Blood levels of superoxide dismutase, malondialdehyde, and glutathione peroxidase were assessed to determine antioxidant capacity and levels of oxidative stress. There were significant group $\times$ time interactions for enzyme activity of $\mathrm{LDH}(\Delta-60 \pm 24.36 \mathrm{U} / \mathrm{L})$ and carotid-toradial pulse wave velocity $(\Delta-1.33 \pm 0.54 \mathrm{~m} / \mathrm{s})$, which significantly decreased $(p<0.05)$ following aspirin supplementation compared to placebo-control. Superoxide dismutase $(\Delta 359 \pm 110 \mathrm{U} / \mathrm{gHb})$ and glutathione peroxidase $(\Delta 28.2 \pm 10.1 \mathrm{U} / \mathrm{gHb})$ significantly decreased while malondialdehyde $(\Delta 3.0 \pm 0.1 \mathrm{mmol} / \mathrm{mL})$ significantly increased $(p<$ $0.05)$ in the placebo-control group compared to the supplementation group. However, there were no changes in lactate concentration levels or augmentation index. These results reveal that low-dose aspirin supplementation would be a useful supplementation therapy to prevent high-intensity exercise training-induced increases in oxidative damage, inflammation, skeletal muscle fatigue, and arterial stiffness in elite Taekwondo athletes.
\end{abstract}

Key words: antioxidant capacity, high-intensity training, NSAID, pulse wave velocity.

\section{Introduction}

Combat sports such as Taekwondo and other martial arts require extremely intense and continuous movement with short rest periods between rounds. These movements necessitate a great amount of anaerobic efforts to change direction, kick, and punch during competition (Campos et al., 2012). Therefore, combat sport athletes typically experience significant fatigue during competition. Fatigue results mainly from increases in metabolic stress-derived oxidative damage and inflammatory molecules (Jammes et al., 2004; Weseler and Bast, 2010) that occur during high-intensity exercise which may hinder both performance and recovery (Duarte et al., 1993). High-intensity exercises such as combat sports increase levels of reactive oxygen species (ROS) production and inflammation (Pal et al., 2018). High levels of ROS and inflammation have been known to result in an attenuation of skeletal

1 - Department of Taekwondo Mission, Kosin University, Busan, South Korea.

2 - School of Health and Kinesiology, University of Nebraska at Omaha, Omaha, NE, USA.

3 - Department of Pathology, Johns Hopkins University School of Medicine, Baltimore, MD, USA. 
muscle function, sports performance, and chronic fatigue, which may collectively cause an increased risk of injury in athletes (Jammes et al., 2004).

Anaerobic metabolism is the primary pathway used during high-intensity exercise to produce ATP, and the enzyme activity of lactate dehydrogenase (LDH) is a critical component for rapid generation of NAD+ from NADH in order to sustain energy production (Gray et al., 2014; Parra-Bonilla et al., 2010). However, chronically elevated enzyme activity of LDH and lactate levels (van Hall, 2010) have been used as markers of muscle cell membrane integrity loss and tissue damage, which may attenuate skeletal muscle function (Jamurtas et al., 2005; Rodrigues et al., 2010). LDH does not have the ability to cross the sarcoplasmic membrane barrier, which contributes to the muscle damage and muscle cell membrane integrity loss following high-intensity exercise (Callegari et al., 2017). Additionally, elevated enzyme activity of LDH is thought to contribute to necrosis or the swelling and rupture of organelles and cell membrane breakdown in the skeletal muscle tissues (Brancaccio et al., 2010; Green et al., 2017). Enzyme activity of LDH can remain elevated above resting levels for up to 72 hours after exercise (Rodrigues et al., 2010), which may further exacerbate loss of muscle cell membrane integrity and tissue damage. Therefore, four weeks of high-intensity training with limited recovery may exacerbate the enzyme activity of LDH and/or exceed the rates of LDH clearance in the muscle. This in turn may attenuate neuromuscular activity due to muscle cell and tissue damage, which may lead to insufficient force generation and poor muscular performance (Callegari et al., 2017; Verma et al., 2016). Thus, the ability to efficiently reduce enzyme activity of LDH and lactate levels posttraining may be crucial to prevent potential muscle damage and improve sports performance.

Although ROS production is critical for cell signaling and is a natural result of ATP production during high-intensity exercise, when produced in excess, ROS causes oxidative stress and induces vascular damage (Thannickal and Fanburg, 2000), especially when ROS production exceeds ROS clearance. It is well documented that high-intensity exercise drastically increases cellular ROS production, which can cause oxidative stress damage in both skeletal muscle and vascular smooth muscle cells (Bessa et al., 2016). Chronically elevated levels of ROS and improper redox balance are thought to contribute to endothelial dysfunction, inflammation, and vascular remodeling (i.e. arterial stiffness) (Bir et al., 2012; Lugrin et al., 2014). If excessive ROS is not properly cleared following high-intensity exercise, oxygen delivery to skeletal muscle can be attenuated, which impairs muscle oxygenation and clearance of metabolic by-products from ATP production that can eventually reduce muscular contraction and sports performance. Additionally, it has been documented that increased enzyme activity of LDH is associated with increased levels of ROS (Mokwatsi et al., 2016), and excessive ROS has also been documented to attenuate muscle cell membrane integrity (Davies et al., 1982). Therefore, proper by-product removal and ROS clearance is crucial for maintaining muscle cell membrane integrity and preventing muscle cell damage.

Superoxide dismutase (SOD) is the first line of the endogenous antioxidant defense system which protects against mitochondrial and NADPH oxidase-produced superoxide (Fukai and Ushio-Fukai, 2011; Michalczyk et al., 2019). In addition to SOD, glutathione peroxidase (GPx) is another endogenous antioxidant enzyme that scavenges hydrogen peroxide (Lubos et al., 2011). Both SOD and GPx are critical for normal cell function and vascular tone. High-intensity exercise has been reported to increase ROS levels and decrease the endogenous antioxidant capacity that results in lipid peroxidation and cell damage, which is indicated by increased levels of malondialdehyde (MDA), a marker of lipid peroxidation (Ayala et al., 2014). Since highintensity exercise has been shown to drastically increase ROS production, and potentially result in oxidative damage, this oxidative damage may also play a role in increasing cellular inflammation (Lugrin et al., 2014). It may be necessary to examine an intervention that protects against the negative effects of excessive exerciseinduced ROS production, increased enzyme activity of LDH, and inflammation, and the utilization of an anti-inflammatory medication may be a viable option (Kotur-Stevuljevic et al., 2007).

Aspirin, also known as acetylsalicylic acid, is a nonsteroidal anti-inflammatory drug 
(NSAID) used for controlling pain, fever, and inflammation (Morris et al., 2009). Due to the pain-relieving effects of aspirin, athletes in combats sports commonly use it during and after training. However, previous studies have suggested that high-dose NSAIDs or prolonged use of NSAIDs can reduce mitochondrial function and vascular function (Mingatto et al., 1996; Moreno-Sanchez et al., 1999), which may impair recovery and exercise performance. However, recent studies have also suggested that NSAIDs can act as a cardioprotective drug (Berger et al., 2006; Morris et al., 2009), reducing the risks of heart disease and stroke when used at a low-dose (75-160 mg) regularly (Dalen, 2006). Additionally, studies have shown that regular dose NSAIDs do not negatively affect mitochondrial function (Uppala et al., 2017), and may in fact increase mitochondrial biogenesis (Kamble et al., 2013). Previous literature demonstrated that aspirin in a low-dose may be beneficial for reducing systemic inflammation without causing harmful effects at the cellular level. To our knowledge, no studies have addressed the impact of low-dose aspirin during high-intensity exercise. Therefore, there is a need to examine the effects of low-dose aspirin supplementation during high-intensity training on muscle fatigue, vascular function, and antioxidant capacity/oxidative stress. Thus, we sought to examine the effects of low-dose aspirin supplementation on the enzyme activity of LDH, lactate concentration, arterial stiffness, antioxidant capacity, and oxidative stress following four weeks of high-intensity exercise training in college-aged, elite male Taekwondo athletes. We hypothesized that aspirin supplementation would have protective effects against high-intensity exercise-induced increases in LDH enzyme activity and lactate concentration, arterial stiffness, and oxidative stress.

\section{Methods}

\section{Participants}

Twenty healthy, elite male Taekwondo athletes (age: $21 \pm 1$ years, BMI: $22.5 \pm 1.5 \mathrm{~kg} / \mathrm{m}^{2}$ ) participated in this study. They were free from diabetes, cancer, cardiovascular, and renal diseases. Participants abstained from caffeinated drinks and alcohol 48-72 hours prior to the pre and post measurements during the study. All participants were restricted from taking any medications or nutraceutical products during the supplementation period. Athletes had no history of using chronic supplementation of antioxidants, aspirin or other NSAIDs in the last 2 years. All participants provided written informed consent, and study protocols were approved by the Institutional Review Board of Kosin University and carried out in accordance with the Declaration of Helsinki. Participant characteristics are presented in Table 1A.

\section{Study Design}

A parallel experimental design was used. After an initial screening, eligible participants were randomly assigned to either the aspirin supplementation group (AS) or the placebocontrolled group (PL). Participants were asked to complete two visits to collect measurements before and after four weeks of supplementation and training. Measurements were collected at the same time of the day ( \pm 1 hour) following an overnight fast. Body height, mass, BMI, and blood pressure were measured, and venous blood samples were taken to measure the enzyme activity of LDH, lactate concentration, antioxidant capacity, and oxidative stress. Arterial stiffness was measured using carotid-to-radial pulse-wave velocity (crPWV) in the carotid-radial segment and the augmentation index (AIx) was assessed in the carotid artery pressure waveform.

\section{Diet and lifestyle control}

The participants' lifestyle was controlled during the study period. The study was conducted at a training camp where food intake, physical activity, and the daily lifestyle were supervised by trained instructors. Participants were instructed not to change their regular caloric intake (maintain $\sim 2.400 \mathrm{kcal}$ per day) during the study. Food logs were obtained throughout the training period to ensure there were no changes in caloric intake. Participants' body mass was measured before and after each training session.

\section{Training program}

High-intensity Taekwondo training was performed five days per week for four weeks. Training intensity was set at $70-90 \%$ heart rate reserve $\left(\mathrm{HRR}=\left[\%\right.\right.$ intensity desired ${ }^{*}\left(\mathrm{HR}_{\max }-\right.$ $\left.\left.H_{\text {rest }}\right]+H_{\text {rest }}\right)$ for the 4 weeks. The heart rate (HR) during training sessions was measured by a Polar RS400 heart rate monitor (Polar Electro, Kempele, Finland). There were four coaches supervising the training sessions, each specifically 
assigned to five participants. The HR monitors were checked every $5 \mathrm{~min}$ during the 100-min training session. If the HR was below or above the training intensity level, participants were encouraged to increase or decrease their effort during the training session.

A training day included $40 \mathrm{~min}$ of kicking, tactical movement, and sparring training, $30 \mathrm{~min}$ of running and circuit training, and $30 \mathrm{~min}$ of weight lifting (Table 1B). All training sessions included various kicking, punching, and tactical movement variations. These variations were performed with a partner. The circuit training component included callisthenic exercises such as push-ups, pull-ups, abdominal exercises, and body weight squats. The weight lifting component included multi-joint movements such as Olympic lifts (clean and jerk, snatch) and core lifts (squat, bench, dead lift).

\section{Supplementation}

The AS group ingested a low-dose (100 mg, Bayer, Leverkusen, Germany) of aspirin, and the PL group ingested a placebo (same appearance flour tablet) daily for four weeks, 30 min after training (Colwell, 2004). On the two non-training days each week, participants arrived to take either aspirin or the placebo at about the same time of day $( \pm 1 \mathrm{~h})$ as they would do so on a training day. The aspirin or the placebo was taken with food and water (500-750 mL) to ensure safety and proper digestion.

\section{Descriptive measurements}

Body height was measured using a standard stadiometer, and body mass was measured using the X-SCAN PLUS II (Jawon Medical, Seoul, South Korea). Participants then rested in an upright seated position for $5 \mathrm{~min}$, and resting brachial BP was measured twice with an automatic sphygmomanometer (Jawon Medical, Seoul, South Korea). The average of the two measurements was recorded for further analysis (Son et al., 2017).

\section{Blood sampling}

Participants were asked to report to the laboratory fully rested and fasted from 8:00 PM the day prior to blood sampling. Venous blood samples $(10 \mathrm{~mL})$ were obtained from an antecubital vein using a vacutainer and a needle before and after the four weeks at the same time of the day $( \pm 1 \mathrm{~h})$. Samples were immediately centrifuged at 3,000 rpm for $10 \mathrm{~min}$ at $4^{\circ} \mathrm{C}$ and stored at $-80^{\circ} \mathrm{C}$ for later analysis.

\section{Lactate dehydrogenase and lactate}

Serum enzyme activity of lactate dehydrogenase (LDH) was determined using an LDH assay kit (Cat. No. ab102526, Abcam, Cambridge, MA) following the manufacturer's instructions. Lactate concentrations were measured using a lactate assay kit (MAK064, Sigma-Aldrich Corp., St. Louis, MO) according to the manufacturer's instructions.

\section{Antioxidant capacity and oxidative stress}

Plasma levels of superoxide dismutase (SOD) were obtained using SOD Assay Kit-WST (Cayman Chemical, Ann Arbor, MI, USA). Samples were mixed with WST working solution and enzyme working solution. The samples were then incubated for $20 \mathrm{~min}$ at 37 . The absorbencies of the incubated samples were acquired at $450 \mathrm{~nm}$ by a plate reader. SOD activity was determined by a Cobas Mira chemistry analyzer (Roche, Basel, Switzerland).

Plasma levels of glutathione peroxidase (GPx) were acquired by a GPx Assay Kit (Cayman Chemical, Ann Arbor, MI, USA). Samples were incubated for $20 \mathrm{~min}$ at 37?. The absorbances of the incubated samples were acquired at $450 \mathrm{~nm}$ by a plate reader. The GPx activity was determined using a Cobas Mira chemistry analyzer (Roche, Basel, Switzerland). The plasma samples were incubated with reagent diluent for 5 $\mathrm{min}$ at room temperature. The incubated samples were stimulated by Ran-Cell total antioxidant control (Randox, Crumlin, County Antrim, UK), and then analyzed at $340 \mathrm{~nm}$ by a plate reader.

Plasma malondialdehyde (MDA) was measured by a BIOXTECHLPO-586 kit (OXIS Health Products, Inc, OR, USA). The plasma samples were incubated with reagent diluent for 5 $\mathrm{min}$ at room temperature. The incubated samples were stimulated by Ran-Cell total antioxidant control (Randox, Crumlin, County Antrim, UK), and then analyzed at $340 \mathrm{~nm}$ by a plate reader.

\section{Arterial stiffness}

Arterial stiffness was tested using arterial tonometry with SphygmoCor (AtCor Medical, New South Wales, Australia) according to the Clinical Application of Arterial Stiffness, Task Force III (Van Bortel et al., 2002). Palpation was used to detect the strongest pulse on both the carotid and radial arteries for tonometer placement. Carotid-to-radial pulse wave velocity 
(crPWV) was measured in the carotid-radial segment using the difference in the carotid-toradial path length $(l)$ divided by the time difference $(\Delta t)$ between pulse arrivals from the carotid artery to the radial artery $(\mathrm{crPWV}=l / \Delta \mathrm{t})$ (Kelly et al., 1989). The augmentation index (AIx) was assessed in the carotid artery pressure waveform. The AIx was calculated by the difference between the forward waveform $\left(P_{1}\right)$ and reflected waveform $\left(P_{2}\right)$ divided by pulse pressure and expressed as a percentage of pulse pressure (Kelly et al., 1989).

\section{Statistical Analysis}

Data were analyzed using a 2 × 2 analysis of variance (ANOVA) with repeated measures (group [AS and PL] * time [before and after 4 weeks]) and Bonferroni adjustments were used to determine the effects of low-dose aspirin on enzyme activity of $\mathrm{LDH}$, lactate concentration, arterial stiffness, antioxidant capacity, oxidative stress, and the AIx. If a significant main effect or interaction was noted, a paired $t$-test was used for post hoc comparisons. Analyses were performed using SPSS 21.0 (IBM SPSS Analytics, Armonk, NY). Data are presented as Mean \pm SD. Statistical significance was set at $p<0.05$. A power analysis calculation determined using $\mathrm{G}^{*}$ Power 3.1 power analysis software (Universität Kiel, Germany) found that a minimum sample size of 20 (10 each group) would allow for the observation of a difference of 3 to $5 \%$ between groups (AS vs. PL) on the primary study outcome variable of crPWV with a power of $80 \%$ (Pietri et al., 2014).

\section{RESULTS}

Participants' characteristics including age, body height, body mass, BMI, and resting BP were not significantly different $(p>0.05)$ between groups (Table 1A). Baseline measurements between the AS and PL groups were not significantly different $(p=0.15)$. There were significant group $x$ time interactions $(p<0.05)$ for LDH (Figure 1A), crPWV (Figure 1C), and SOD, GPx, and MDA (Table 1C). Enzyme activity of LDH and crPWV significantly decreased following training in the AS group $(p<0.05)$, but showed no significant change in the PL group. After 4 weeks, SOD and GPx both significantly decreased $(p<0.05)$ in the PL group compared to the AS group. Additionally, MDA significantly increased $(p<0.05)$ in the PL group, but not in the AS group following supplementation and training. There was no significant change ( $p=$ 0.21) in lactate (Figure 1B) concentrations or the AIx between AS and PL groups following four weeks of supplementation and training.

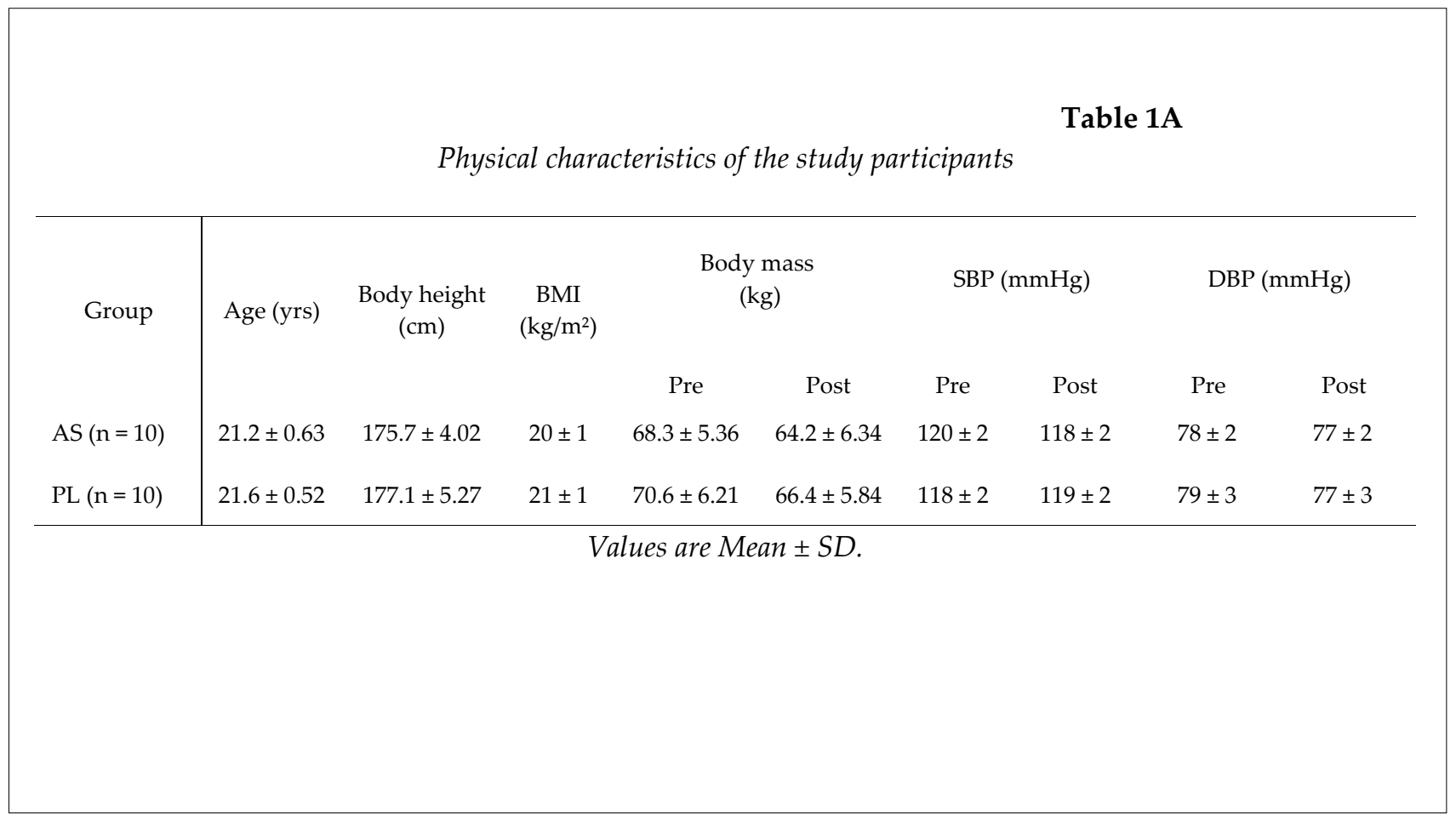


Table 1B

Daily Taekwondo training program

\begin{tabular}{|c|c|c|}
\hline Order & Exercise & Intensity \\
\hline $\begin{array}{l}\text { Warm-up } \\
\text { (10 min) }\end{array}$ & & - \\
\hline $\begin{array}{c}\text { Training } \\
\text { (100 min) }\end{array}$ & $\begin{array}{c}\text {-Tube kicks } \\
\cdot \text { Kicks } \\
\text {-Tactical kicking } \\
\text { ·Forward and backward steps } \\
\text {-Step sparring } \\
\text {-Sparring } \\
\text {-Running } \\
\text { - Circuit training } \\
\text {-Weight lifting }\end{array}$ & $\begin{array}{c}70-90 \% \\
\text { HRR } \\
(159-185 \mathrm{bpm})\end{array}$ \\
\hline $\begin{array}{c}\text { Cool-down } \\
\text { (10 min) }\end{array}$ & Stretching & - \\
\hline
\end{tabular}

Table 1C

Levels of superoxide dismutase (SOD), malondialdehyde (MDA), and glutathione peroxidase (GPx) before and after the Taekwondo training program

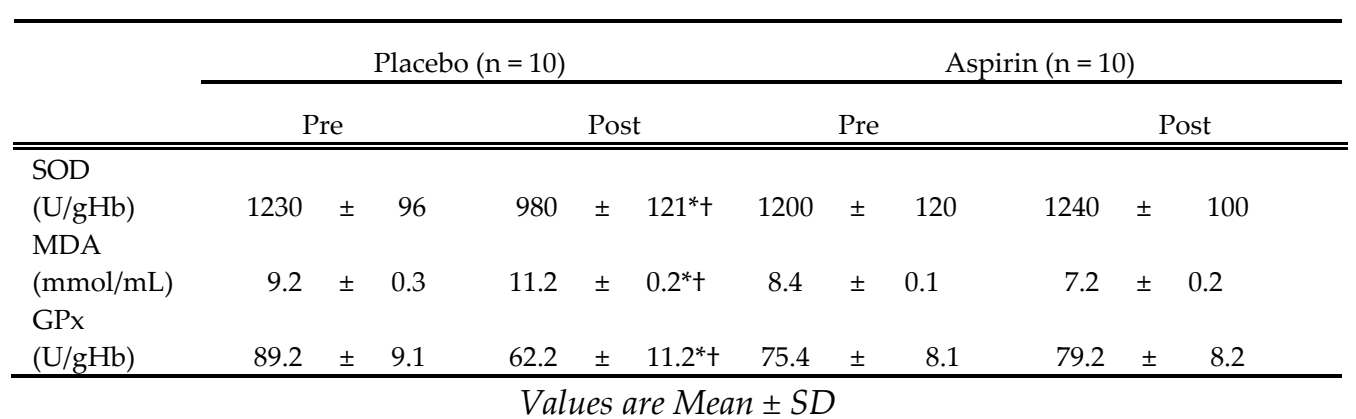


Figure 1A

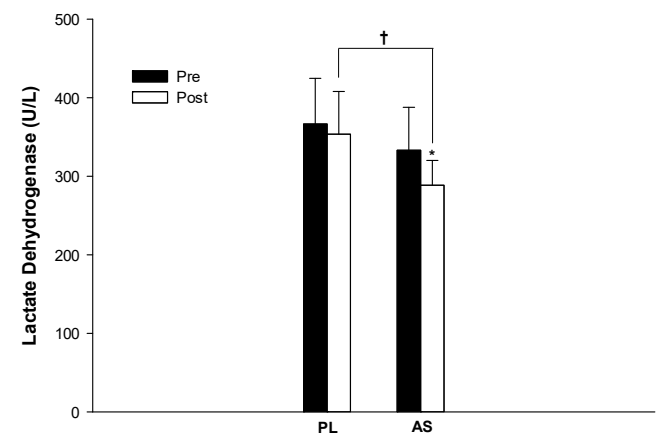

Enzyme activity of lactate dehydrogenase $(\mathrm{U} / \mathrm{L})$ pre-and post-supplementation between the aspirin (AS) and placebo (PL) groups: Post lactate dehydrogenase (LDH) enzyme activity decreased in the AS group and did not change in the PL group. Additionally, LDH enzyme activity was lower in the post AS group compared to the post PL group.

Values are Mean $\pm S D ;{ }^{*} p<0.05$ vs. Pre; $+p<0.05$ vs. PL

Figure 1B

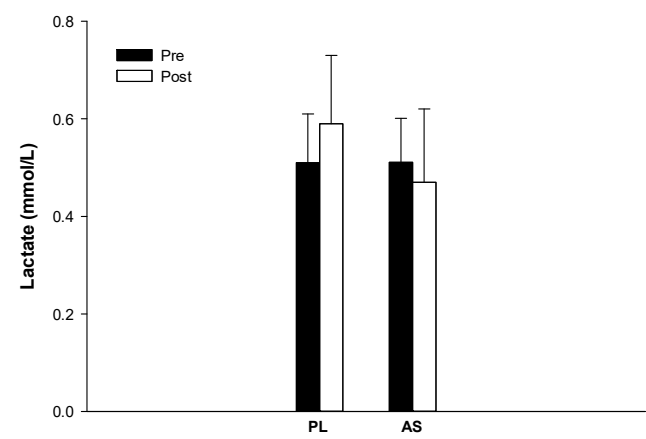

Changes in lactate concentration $(\mathrm{mmol} / \mathrm{L})$ pre-and post-supplementation between the aspirin (AS) and placebo (PL) groups: No significant difference in lactate concentration was seen in aspirin or placebo supplementation in the AS or PL groups.

Values are Mean $\pm S D$.

Figure 1C

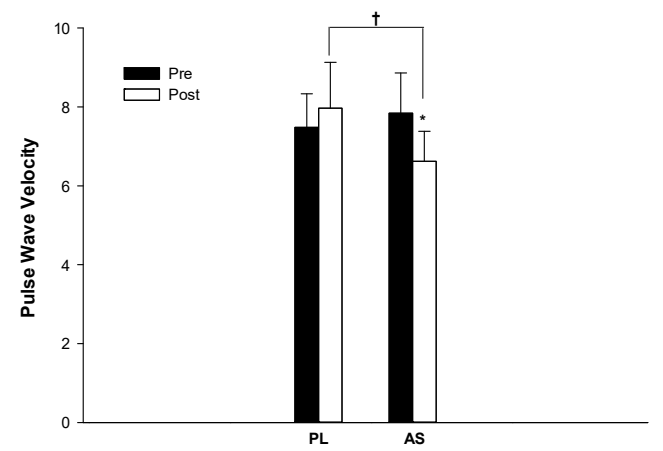

Changes in carotid-to-radial pulse wave velocity $(\mathrm{m} / \mathrm{s})$ pre-and post-supplementation between the aspirin (AS) and placebo (PL) groups: Post carotid-to-radial pulse wave velocity (crPWV) decreased in the AS group and showed a trend to be higher in the PL group. Also, crPWV was lower in the post AS group compared to the post PL group.

Values are Mean $\pm S D ;{ }^{*} p<0.05$ vs. Pre; $t p<0.05$ vs. PL 


\section{Discussion}

The purpose of this study was to examine the effects of four weeks of aspirin supplementation on blood levels of enzyme activity of $\mathrm{LDH}$, lactate concentration, arterial stiffness, antioxidant capacity, and oxidative stress during high-intensity exercise in elite Taekwondo athletes. There were a number of novel findings. First, enzyme activity of LDH was significantly reduced following aspirin supplementation (Figures 1A). Second, aspirin supplementation reduced crPWV, which implies that aspirin may be a useful pharmaceutical aid to protect against the negative effects of highintensity exercise on arterial function (Figure 1C). Finally, antioxidant capacity and oxidative stress levels were maintained in the AS group, but antioxidant capacity was significantly reduced and oxidative stress was significantly increased in the PL group (Table 1C). These findings reveal that aspirin supplementation may reduce highintensity exercise-induced skeletal muscle damage and oxidative stress by reducing the enzyme activity of $\mathrm{LDH}$ and improving both antioxidant capacity and ROS clearance. Additionally, aspirin may also have protective effects against highintensity exercise-induced arterial stiffness.

Aspirin reduces low-grades of systemic inflammation (Morris et al., 2009). Fatigue or the attenuation of body functionality can be evoked by low-grades of systemic inflammation, metabolic by-products, and accumulation of $\mathrm{H}^{+}$ ions, increased enzyme activity of $\mathrm{LDH}$, and elevated lactate that are induced by high-intensity exercise (Louati and Berenbaum, 2015; Rodrigues et al., 2010; van Hall, 2010). Anaerobic metabolism is the most dominant energy system during highintensity exercise. It includes repeated movements with a high muscle force production and typically results in excessive amounts of $\mathrm{H}^{+}$ions, increased enzyme activity of LDH, and increased lactate (Karamizrak et al., 1994; Rodrigues et al., 2010). High-intensity training seen in Taekwondo primarily depends on anaerobic metabolism (Campos et al., 2012), which leads to elevated amounts of anaerobic metabolic stress and systemic inflammation that may result in attenuated muscular contraction and poor muscular contractile performance. Therefore, the anti-inflammatory properties of aspirin may play a role in reducing fatigue due to exercise.
However, inflammatory markers were not directly examined in this study and further investigation is warranted.

$\mathrm{LDH}$ is a critical enzyme for ATP production via $\mathrm{NADH} / \mathrm{NAD}^{+}$-mediated anaerobic energy metabolism (Gray et al., 2014; ParraBonilla et al., 2010). Additionally, the enzyme activity of LDH has been used as a biomarker of muscle damage, and the enzyme activity is typically elevated after exercise (Rodrigues et al., 2010; van Hall, 2010). Previous studies have suggested that increased enzyme activity of LDH in the blood negatively affects $\mathrm{pH}$, cellular ATP production, muscle cell membrane integrity, and is associated with increased levels of ROS production that can further exacerbate cellular damage (Callegari et al., 2017; Lippi et al., 2008; Mokwatsi et al., 2016). Furthermore, decreased muscle cell and mitochondrial membrane integrity has been known to increase ROS production due to the attenuated membrane potential and greater proton leak (Park et al., 2014, 2018). One of the novel findings of this study is that the enzyme activity of LDH was significantly decreased following four weeks of aspirin supplementation during high-intensity training. A previous study reported normal resting enzyme activity of LDH to be approximately $243.4 \pm 43.5$ U/L (Kanter et al., 1988). Following aspirin supplementation, the AS group went from $333.3 \pm$ $54.56 \mathrm{U} / \mathrm{L}$ to $288.7 \pm 31.71 \mathrm{U} / \mathrm{L}$, suggesting that aspirin may help maintain normal enzyme activity of LDH during training and/or may help with clearance of LDH. However, the PL group had elevated enzyme activity of LDH above this normal range. Exercise is known to increase LDH enzyme activity, as a previous study reported that high-intensity sprint training significantly increased the enzyme activity of LDH in collegiate soccer players, and it remained elevated for 72 hours post-exercise (Verma et al., 2016). The elevated LDH enzyme activity could negatively affect speed, agility, and power in athletes (Verma et al., 2016). The Taekwondo training program in this study consisted of high-intensity exercise, and our preliminary data (unpublished) showed that enzyme activity of LDH was elevated after one acute bout of exercise training at the same intensity used in this study. This result helped us speculate that the four weeks of high-intensity training without adequate recovery would cause 
chronically elevated enzyme activity and poor clearance of LDH in these athletes, and that this long-term increase in enzyme activity of LDH may result in muscle cell membrane and tissue damage (Brancaccio et al., 2010; Callegari et al., 2017). Taekwondo requires high levels of speed, agility, and power, and chronically elevated enzyme activity of LDH may attenuate athletic performance. Considering that the AS group had significantly decreased enzyme activity of LDH, aspirin may be a beneficial supplement for Taekwondo athletes to reduce training-induced muscle cell membrane damage, muscle cell membrane integrity, tissue damage, and excessive ROS accumulation as a result of high-intensity training (Brancaccio et al., 2010; Mokwatsi et al., 2016). This may be particularly relevant for inseason training, especially if athletes train at high intensity on a daily basis with inadequate time for recovery. However, the mechanism(s) underlying aspirin supplementation and reduced enzyme activity of LDH warrant further study.

It is well known that lactate concentrations increase during exercise, resulting in decreased muscle $\mathrm{pH}$. High concentrations of lactate have also been used as a marker of fatigue (Hultman et al., 1986). Interestingly, post measurements of blood lactate levels did not differ between the AS and PL groups in this study. This may be because our participants were at the elite level and had several years of training experience. It has been reported that highlytrained and elite-level athletes have a greater postexercise lactate removal capacity (Favier et al., 1986). It is possible that these elite-level Taekwondo athletes already had a sufficient lactate removal capacity. Therefore, they have better efficiency for lactate clearance during training.

In addition to the increase in enzyme activity of LDH and lactate concentrations, highintensity exercise also produces substantial amounts of ROS, which may ultimately lead to oxidative stress (Weseler and Bast, 2010). Low levels of ROS are required for cell signaling, muscular adaptations, and energy production, but exposure to chronically elevated ROS can cause muscle contractile dysfunction, fatigue, and cardiovascular dysfunction in athletes (Anh et al., 2019; Cortez-Cooper et al., 2005). The effects of ROS depend on the individual athlete's training level; although, when exercise intensity is high without proper recovery time, ROS can cause muscle contractile dysfunction that can attenuate muscular performance even in highly trained populations (Michalczyk et al., 2015; Ahn et al., 2019). Two antioxidant capacity markers, SOD and GPx, were both significantly reduced in the PL group after four weeks of high-intensity training, while the AS group showed no significant change. MDA, a marker of lipid peroxidation and oxidative stress (Ayala et al., 2014), was significantly increased in the PL group after the training program, but there was no change in the AS group. These findings reveal that aspirin supplementation was beneficial for maintaining antioxidant capacity while reducing oxidative stress in the AS group. Increased antioxidant capacity would allow for better clearance of ROS, likely resulting in both reduced cellular damage and beneficial effects on skeletal muscle performance. Additionally, the improved antioxidant capacity from aspirin supplementation may result in less $\mathrm{H}^{+}$ion and free radical accumulation, which may reduce the acidity in the working skeletal muscle, allowing for improved muscle function during training.

Blood pressure is regulated in-part by arterial smooth muscle tone (Touyz et al., 2018). Arterial smooth muscle tone is determined by the arterial elasticity, smooth muscle contractility, and endothelial function within the artery (Brozovich et al., 2016). As blood pressure rises, recruitment of elastic collagen fibers in the arterial smooth muscle increases (Quinn et al., 2012). Chronically increased blood pressure can result in increased levels of arterial stiffness (Quinn et al., 2012). The AIx and crPWV are clinical measurements of arterial stiffness that have been used to determine the structural and functional health of the vasculature (McEniery et al., 2006). Arterial smooth muscle contractility/elasticity and crPWV are inversely related, therefore a higher crPWV accompanied by decreased arterial elasticity results in higher arterial stiffness, and a higher crPWV has been shown to be an indicator of atherosclerotic risk (Park et al., 2006). When crPWV is lower, arterial structure and function are at a more optimal level. Proper arterial function and compliance are important for both blood pressure regulation and oxygen delivery capacity (Tomiyama and Yamashina, 2010), and 
this is particularly important during exercise.

During high-intensity exercise, blood pressure and mean arterial pressure rise and both contribute to arterial stiffness (Miyachi et al., 2004). The increase in systolic blood pressure specifically during high-intensity exercise may further contribute to increased arterial stiffness (Cortez-Cooper et al., 2005; Miyachi et al., 2004). The PL group in this study showed a tendency of increased arterial stiffness, indicating that highintensity training may have detrimental effects on arterial compliance, which can impair blood flow and vascular function. In contrast to the PL group, we found that low-dose aspirin supplementation demonstrates protective effects against the high-intensity exercise-induced increases in arterial stiffness. To our knowledge, this is the first study to examine the beneficial effects of aspirin supplementation on highintensity exercise training and arterial stiffness. However, we do not have clear evidence regarding the mechanism(s) responsible for the reduced arterial stiffness in the AS group. Nevertheless, we found that aspirin supplementation may play a role in reducing exercise-induced inflammation and oxidative stress, both of which are thought to be key players that cause damage to the vascular endothelial cells and smooth muscle cells, therefore they may play a role in reducing levels of arterial stiffness. These mechanisms underlying the effect(s) of aspirin on reducing arterial stiffness warrant further research.

The AIx is another commonly utilized clinical measurement of arterial stiffness, which is derived from the ascending aortic pressure waveform (Kelly et al., 1989). Unlike crPWV, the AIx was not significantly changed following four weeks of aspirin supplementation with highintensity exercise. One possible explanation for this is that the AIx is mainly influenced by the heart rate (HR), meaning that changes in the HR are critical for determining the AIx (Wilkinson et al., 2000). Participants in both groups were similar in age and training status, which indicates that they may have had similar resting HRs and HR responses to the exercise intensity, and thus may not have experienced noticeable differences in the AIx. Additionally, all participants were relatively young and healthy; therefore, the beneficial effects of aspirin on the AIx may be masked in those with already-healthy AIx measures (Chung et al., 2010).

Our findings demonstrate that four weeks of high-intensity exercise with low-dose aspirin supplementation reduces the enzyme activity of $\mathrm{LDH}$, reduces arterial stiffness, and helps maintain proper systemic redox balance. Aspirin has typically been used to reduce exerciseinduced pain, muscle soreness, and minor levels of inflammation. However, these results show that aspirin can also provide beneficial effects on exercise performance and recovery by 1) limiting exercise-induced muscle cell membrane and tissue damage by improving LDH and ROS clearance, and 2) by increasing oxygen delivery capacity to the skeletal muscle through reduced arterial stiffness following chronic high-intensity exercise. Therefore, aspirin supplementation during high-intensity training with limited time for recovery may be useful for improving exercise performance and recovery in elite Taekwondo athletes and in athletes of other combat sports.

\section{Acknowledgements}

No financial or material support of any kind was received for the work described in this article. We are grateful for our participants.

\section{References}

Anh B, Ranjit R, Premkumar P, Pharaoh G, Pierkarz KM, Matsuzaki S, Claflin DR, Riddle K, Judge J, Bhaskaran S, Satara Natarajan K, Barboza E, Wronowski B, Kinter M, Humphries KM, Griffin TM, Freeman WM, Richardson A, Brooks SV, Van Remmen H. Mitochondrial oxidative stress impairs contractile function but paradoxically increases muscle mass via fiber branching. J Cachexia Sarcopenia Muscle, 2019; 10: 411-428 
Ayala A, Munoz MF, Arguelles S. Lipid peroxidation: production, metabolism, and signaling mechanisms of malondialdehyde and 4-hydroxy-2-nonenal. Oxid Med Cell Longev, 2014; 2014: 1-31

Berger JS, Roncaglioni MC, Avanzini F, Pangrazzi I, Tognoni G, Brown DL. Aspirin for the primary prevention of cardiovascular events in women and men: a sex-specific meta-analysis of randomized controlled trials. Jama-J Am Med Assoc, 2006; 295: 306-313

Bessa AL, Oliveira VN, Agostini GG, Oliveira RJ, Oliveira AC, White GE, Wells GD, Teixeira DN, Espindola FS. Exercise intensity and recovery: biomarkers of injury, inflammation, and oxidative stress. $J$ Strength Cond Res, 2016; 30: 311-9

Bir SC, Kolluru GK, Fang K, Kevil CG. Redox balance dynamically regulates vascular growth and remodeling. Semin Cell Dev Biol, 2012; 23: 745-57

Brancaccio P, Lippi G, Maffulli N. Biochemical markers of muscular damage. Clin Chem Lab Med, 2010; 48: 757-67

Brozovich FV, Nicholson CJ, Degen CV, Gao YZ, Aggarwal M, Morgan KG. Mechanisms of vascular smooth muscle contraction and the basis for pharmacologic treatment of smooth muscle disorders. Pharmacol Rev, 2016; 68: 476-532

Callegari GA, Novaes JS, Neto GR, Dias I, Garrido ND, Dani C. Creatine kinase and lactate dehydrogenase responses after different resistance and aerobic exercise protocols. J Hum Kinet, 2017; 58: 65-72

Campos FAD, Bertuzzi R, Dourado AC, Santos VGF, Franchini E. Energy demands in taekwondo athletes during combat simulation. Eur J Appl Physiol, 2012; 112: 1221-28

Chung JW, Lee, YS, Kim JH, Seong MJ, Kim SY, Lee JB, Ryu JK, Choi JY, Kim KS, Chang SG, Lee GH, Kim $\mathrm{SH}$. Reference values for the augmentation index and pulse pressure in apparently healthy korean subjects. Korean Circ J, 2010; 40: 165-71

Colwell JA. Aspirin therapy in diabetes. Diabetes Care, 2004; 27: 72-73

Cortez-Cooper MY, DeVan AE, Anton MM, Farrar RP, Beckwith KA, Todd JS, Tanaka A. Effects of high intensity resistance training on arterial stiffness and wave reflection in women. Am J Hypertens, 2005; 18: $930-34$

Dalen JE. Aspirin to prevent heart attack and stroke: What's the right dose?. Am J Med, 2006; 119: 198-202

Davies KJ, Quintanilha AT, Brooks GA, Packer LF. Free radicals and tissue damage produced by exercise. Biochem Bioph Res Co, 1982; 107: 1198-205

Duarte JA, Appell HJ, Carvalho F, Bastos ML, Soares JM. Endothelium-derived oxidative stress may contribute to exercise-induced muscle mamage. Int J Sports Med, 1993; 14: 440-43

Favier RJ, Constable SH, Chen M, Holloszy JO. Endurance exercise training reduces lactate production. $J$ Appl Physiol 1986; 61: 885-9

Fukai T, Ushio-Fukai M. Superoxide dismutases: role in redox signaling, vascular function, and diseases. Antioxid Redox Sign, 2011; 15:1583-606

Gray LR, Tompkins SC, Taylor EB. Regulation of pyruvate metabolism and human disease. Cell Mol Life Sci, 2014; 71: 2577-604

Green H, Tobar A, Gafter-Gvili A, Leibovici L, Klein T, Rahamimov R, Mor E, Grossman A. Serum lactate dehydrogenase is elevated in ischemic acute tubular necrosis but not in acute rejection in kidney transplant patients. Prog Transplant, 2017; 27: 53-57

Hultman E, Spriet LL, Soderlund K. Biochemistry of muscle fatigue. Biochim Biophys Acta, 1986; 45: 97-106

Jammes Y, Steinberg JG, Bregeon F, Delliaux S. The oxidative stress in response to routine incremental cycling exercise in healthy sedentary subjects. Resp Physiol Neurobi, 2004; 144: 81-90

Jamurtas AZ, Theocharis V, Tofas T, Tsiokanos A, Yfanti C, Paschalis V, Koutedakis Y, Nosaka K. Comparison between leg and arm eccentric exercises of the same relative intensity on indices of muscle damage. Eur J Appl Physiol, 2005; 95: 179-85

Kamble PK, Selvarajan K, Aluganti Narasimhulu C, Nandave M, Parthasarathy S. Aspirin may promote mitochondrial biogenesis via the production of hydrogen peroxide and the induction of Sirtuin1/PGC1alpha genes. Eur J Pharmacol, 2013; 699: 55-61

Kanter MM, Lesmes GR, Kaminsky LA, La Ham-Saeger J, Nequin ND. Serum creatine kinase and lactate dehydrogenase changes following an eighty kilometer race. Relationship to lipid peroxidation. Eur J Appl Physiol O, 1988; 57: 60-3 
Karamizrak SO, Ergen E, Tore IR Akgun N. Changes in serum creatine kinase, lactate dehydrogenase and aldolase activities following supramaximal exercise in athletes. J Sport Med Phys Fit, 1994; 34: 141-6

Kelly R, Hayward C, Avolio A, O'Rourke M. Noninvasive determination of age-related changes in the human arterial pulse. Circulation, 1989; 80: 1652-59

Kotur-Stevuljevic J, Memon L, Stefanovic A, Spasic S, Spasojevic-Kalimanovska V, Bogavac-Stanojevic N, Kalimanovska-Ostric D, Jelic-Ivanovic Z, Zunic G. Correlation of oxidative stress parameters and inflammatory markers in coronary artery disease patients. Clin Biochem, 2007; 40:181-7

Lippi G, Schena F, Salvagno GL, Montagnana M, Gelati M, Tarperi C, Banfi G, Guidi GC. Acute variation of biochemical markers of muscle damage following a 21-km, half-marathon run. Scand J Clin Lab Inv, 2008; 68: 667-72

Louati K, Berenbaum F. Fatigue in chronic inflammation - a link to pain pathways. Arthritis Res Ther, 2015; 17: 254

Lubos E, Loscalzo J, Handy DE. Glutathione peroxidase-1 in health and disease: from molecular mechanisms to therapeutic opportunities. Antioxid Redox Sign, 2011; 15:1957-97

Lugrin J, Rosenblatt-Velin N, Parapanov R, Liaudet, L. The role of oxidative stress during inflammatory processes. Biol Chem, 2014; 395: 203-30

McEniery CM, Wallace S, Mackenzie IS, McDonnell B, Yasmin, Newby DE, Cockcroft JR, Wilkinson IB. Endothelial function is associated with pulse pressure, pulse wave velocity, and augmentation index in healthy humans. Hypertension, 2006; 48: 602-8

Michalczyk M, Poprzęcki S, Czuba M, Zydek G, Jagsz S, Sadowska-Krępa E, Zając A. Blood antioxidant status in road cyclists during progressive (VO2max) and constant cyclist intensity test (MLSS). J Sports Med Phys Fitness. 2015; 55(9): 855-64

Michalczyk, M.M., Chycki, J., Zajac, A. Petr M, Czuba M, Langfort J. Three weeks of intermittent hypoxic training affect antioxidant enzyme activity and increases lipid peroxidation in cyclists. Monatsh Chem 2019; 150: 1703-1710

Mingatto FE, Santos AC, Uyemura SA, Jordani MC, Curti C. In vitro interaction of nonsteroidal antiinflammatory drugs on oxidative phosphorylation of rat kidney mitochondria: respiration and ATP synthesis. Arch Biochem Biophys, 1996; 334: 303-8

Miyachi M, Kawano H, Sugwara J, Takahashi K, Hayashi K, Yamazaki K, Tabata I, Tanaka H. Unfavorable effects of resistance training on central arterial compliance: a randomized intervention study. Circulation, 2004; 110: 2858-63

Moreno-Sanchez R, Bravo C, Vasquez C, Ayala G, Silveira LH, Martinez-Lavin M. Inhibition and uncoupling of oxidative phosphorylation by nonsteroidal anti-inflammatory drugs: study in mitochondria, submitochondrial particles, cells, and whole heart. Biochem Pharmacol, 1999; 57: 743-52

Morris T, Stables M, Hobbs A, de Souza P, Colville-Nash P, Warner T, Newson J, Bellingan G, Gilroy DW. Effects of Low-Dose Aspirin on Acute Inflammatory Responses in Humans. J Immunol, 2009; 183: 208996

Mokwatsi GG, Schutte AE, Kruger R. A biomarker of tissue damage, lactate dehydrogenase, is associated with fibulin-1 and oxidative stress in blacks: the SAfrEIC study. Biomarkers, 2016; 21: 48-55

Pal S, Chaki B, Chattopadhyay S, Bandyopadhyay A. High-Intensity Exercise Induced Oxidative Stress and Skeletal Muscle Damage in Postpubertal Boys and Girls: A Comparative Study. J Strength Cond Res, 2018; 32: 1045-52

Parra-Bonilla G, Alvarez DF, Al-Mehdi AB, Alexeyev M, Stevens T. Critical role for lactate dehydrogenase A in aerobic glycolysis that sustains pulmonary microvascular endothelial cell proliferation. $\mathrm{Am} J$ Physiol-Lung C, 2010; 299: L513-22

Park SU, Jung WS, Moon SK, Ko CN, Cho KH, Kim YS, Bae HS. Chunghyul-Dan (Qingxie-Dan) improves arterial stiffness in patients with increased baPWV. Am J Chinese Med, 2006; 34: 553-63

Park SY, Gifford JR, Andtbacka RH, Trinity JD, Hyngstrom JR, Garten RS, Diakos NA, Ives SJ, Dela F, Larsen S, Drako S, Richardson RS. Cardiac, skeletal, and smooth muscle mitochondrial respiration: are all mitochondria created equal?. Am J Physiol-Heart C, 2014; 307: H346-52 
Park SY, Kwon OS, Andtbacka RHI, Hyngstrom JR, Reese V, Murphy MP, Richardson RS. Age-related endothelial dysfunction in human skeletal muscle feed arteries: the role of free radicals derived from mitochondria in the vasculature. Acta Physiol, 2018; 222

Pietri P, Vlachopoulos C, Terentes-Printzios D, Xaplanteris P, Aznaouridis K, Petrocheilou K, Stefanadis C. Beneficial effects of low-dose aspirin on aortic stiffness in hypertensive patients. Vasc Med, 2014; 19: $452-7$

Quinn U, Tomlinson LA, Cockcroft JR. Arterial stiffness. J Roy Soc Med, 2012; 1

Rodrigues BM, Dantas E, de Salles BF, Miranda H, Koch AJ, Willardson JM, Simao R. Creatine kinase and lactate dehydrogenase responses after upper-body resistance exercise with different rest intervals. $J$ Strength Cond Res, 2010; 24: 1657-62

Son WK, Sung KD, Cho JM, Park SY. Combined exercise reduces arterial stiffness, blood pressure, and blood markers for cardiovascular risk in postmenopausal women with hypertension. Menopause, 2017; 24: $262-68$

Thannickal VJ, Fanburg BL. Reactive oxygen species in cell signaling. Am J Physiol-Lung C, 2000; 279: 1005-28

Tomiyama H, Yamishina A. Non-invasive vascular function tests: their pathophysiological background and clinical application. Circulation J, 2010; 74: 24-33

Touyz RM, Alves-Lopes R, Rios FJ, Camargo LL, Anagnostopoulou A, Arner A, Montezano AC. Vascular smooth muscle contraction in hypertension. Cardiovasc Res, 2018; 114: 529-39

Uppala R, Dudiak B, Beck ME, Bharathi SS, Zhang Y, Stolz DB, Goetzman ES. Aspirin increases mitochondrial fatty acid oxidation. Biochem Bioph Res Co, 2017; 482: 346-51

Van Bortel LM, Duprez D, Starmans-Kool MJ, Safar ME, Giannattasio C, Cockcroft J, Kaiser DR, Thuillez C. Clinical applications of arterial stiffness, Task Force III: recommendations for user procedures. Am J Hypertens, 2002; 15: 445-52

van Hall G. Lactate kinetics in human tissues at rest and during exercise. Acta Physiol (Oxf), 2010; 199: 499508

Verma S, Moiz JA, Shareef MY, Husain ME. Physical performance and markers of muscle damage following sport-specific sprints in male collegiate soccer players: repeated bout effect. J Sport Med Phys Fit, 2016; 56: 765-74

Weseler AR, Bast A. Oxidative stress and vascular function: implications for pharmacologic treatments. Curr Hypertens Rep, 2010; 12: 154-61

Wilkinson IB, MacCallum H, Flint L, Cockcroft JR, Newby DE, Webb DJ. The influence of heart rate on augmentation index and central arterial pressure in humans. J Physiol, 2000; $525 \mathrm{Pt}$ 1: 263-70

\section{Corresponding author:}

\section{Song-Young Park, Ph.D.}

University of Nebraska at Omaha

6001 Dodge Street, Omaha, Nebraska, USA

Phone: (402) 554-3374

Email: song-youngpark@unomaha.edu 